Technical Note

\title{
Coupled thermocapillary convection on Marangoni convection in liquid layers with curved free surface
}

\author{
K. Li *, Z.M. Tang, W.R. Hu \\ Key Laboratory of Microgravity, Institute of Mechanics, CAS, Beijing 100190, China
}

\section{A R T I C L E I N F O}

\section{Article history:}

Received 15 August 2011

Received in revised form 7 December 2011

Accepted 15 December 2011

Available online 16 January 2012

\section{Keywords:}

Liquid layer

Large Prandtl number

Pearson instability

Thermocapillary flow

Marangoni flow

\begin{abstract}
A B S T R A C T
This paper numerically studied the coupled Marangoni convection and thermocapillary convection in a finite liquid layer $(\operatorname{Pr}=11.6)$ in the microgravity conditions. The multi-cellular flow structure and the marginal instability boundary of the coupled convection are predicted. Oscillatory coupled convection is also reported in concave liquid layers of volume ratio between 0.80 and 0.85 .
\end{abstract}

(c) 2012 Elsevier Ltd. All rights reserved.

\section{Introduction}

Pearson's linear stability analysis indicated that Marangoni convection occurs in a bottom-heated infinite liquid layer only when the vertical applied temperature difference exceeds a threshold [1]. Most related studies assumed a planar free surface and focused on the aspect-ratio dependent multi-cellular flow structure, e.g. $[2,3]$. On the other hand, thermocapillary convection occurs as long as a lateral applied temperature difference appears. It influences the Marangoni convection regimes when the convections are coupled such as in a plane liquid layer with an inclined temperature gradient, e.g. [4,5]. Transition among different flow patterns occurs when the lateral temperature gradient changes. Note that the planar free-surface assumption is not always valid in the microgravity conditions. Even if the applied temperature difference is strictly perpendicular to the liquid layer, the concurrent lateral temperature gradient due to the curved free surface results in the coupled Marangoni convection and thermocapillary convection in the microgravity conditions.

This paper numerically studied the coupled Marangoni convection and thermocapillary convection in a finite liquid layer $(P r=11.6)$ in the microgravity conditions. The influences of the volume ratio and Prandtl number of the liquid layer on the multi-cellular flow structure and the marginal instability boundary of the coupled convection are studied in detail.

\footnotetext{
* Corresponding author. Tel.: +86 10 82544098; fax: +86 1082544096 .

E-mail address: likai@imech.ac.cn (K. Li).
}

\section{Physical model and mathematical formulations}

Fig. 1 shows the 2D models of finite liquid layers with curved free surface ( $1 \mathrm{cSt}$ silicone oil with $\operatorname{Pr}=11.6$ ). The depths of the liquid layer at the sidewalls and central line are $h_{0}=3 \mathrm{~mm}$ and $h_{c}$ respectively. The extension of liquid layer is $l=55.5 \mathrm{~mm}$. The free surface configuration is $h(x)$. The ambient temperature $T_{e}$ is $25^{\circ} \mathrm{C}$. Both sidewalls are assumed to be thermally isolated, impermeable and friction-less, the bottom is heated to an expected temperature $T_{b}$. The liquid is assumed to be incompressible with constant viscosity and thermal diffusivity. The surface tension gradient on the temperature at the free surface $\left(\left|\sigma_{T}\right|\right)$ is assumed to be constant. $U_{0}=\left|\sigma_{T}\right| \Delta T / \rho v, h_{0}$ and the pressure $\rho v U_{0} / h_{0}$ are respectively the characteristic scales for the velocity, length and pressure, where $\rho$ is the density of the fluid and $v$ the kinematic viscosity. The temperature with respect to $T_{e}$ is scaled by $\Delta T=T_{b}-T_{e}$. With the non-dimensional vorticity $\omega$ and stream function $\psi$,

$\nabla \times \psi=V, \quad \nabla \times \nabla \times \psi=\omega$

the non-dimensional governing equations can be expressed as follows:

$\frac{\partial \omega}{\partial t}+\mathbf{V} \cdot \nabla \omega-\omega \cdot \nabla V=\frac{P r}{M a} \nabla^{2} \omega$

$\frac{\partial T^{*}}{\partial t}+\mathbf{V} \cdot \nabla T^{*}=\frac{1}{M a} \nabla^{2} T^{*}$

Here $\operatorname{Pr}=v / \kappa$ is the Prandtl number, $M a=\left|\sigma_{T}\right| \Delta T h_{0} / \rho v \kappa$ the Marangoni number, $v$ the thermal diffusivity and $\kappa$ the thermal dif- 
(a)

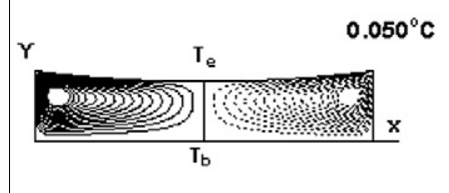

$0.130^{\circ} \mathrm{C}$

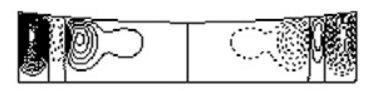

$0.150^{\circ} \mathrm{C}$

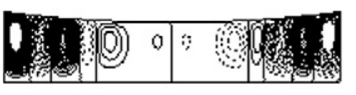

$0.160^{\circ} \mathrm{C}$

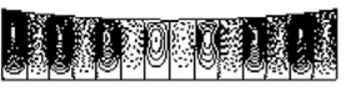

(b)

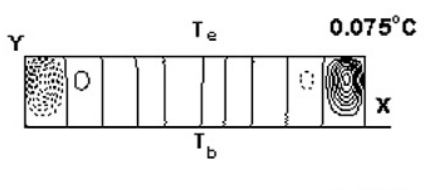

$0.130^{\circ} \mathrm{C}$

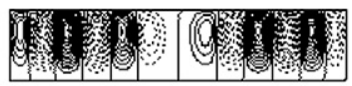

$0.133^{\circ} \mathrm{C}$

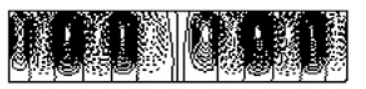

$0.135^{\circ} \mathrm{C}$

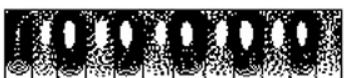

(c)
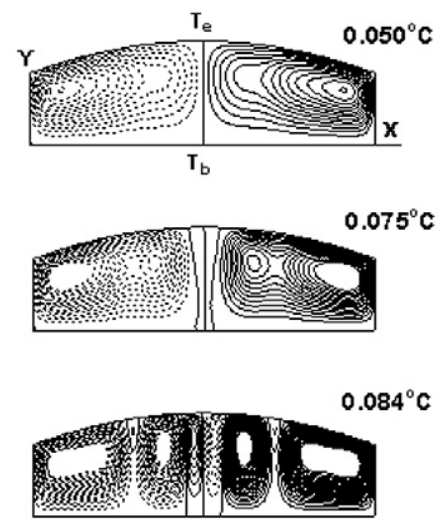

$0.085^{\circ} \mathrm{C}$

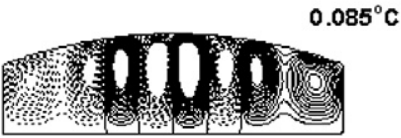

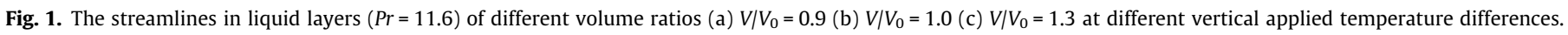

fusivity. The effective Marangoni number $B=\left|\sigma_{T}\right|\left(T_{b}-T_{c i}\right) h_{0} / \rho v \kappa$ is also defined where $T_{c i}$ is the temperature at the central point of free surface. The equation of mass conservation is automatically satisfied.

The boundary conditions are as follows:

$x=0 \quad$ and $\quad x=\frac{l}{h_{0}}: \psi=0, \quad \omega=\frac{\partial^{2} \psi}{\partial x^{2}}=0, \quad \frac{\partial T^{*}}{\partial x}=0$

$y=0: \psi=0, \quad \frac{\partial \psi}{\partial x}=0, \quad \omega=-\frac{\partial^{2} \psi}{\partial y^{2}}, \quad T^{*}=T_{b}^{*}$

At the free surface $y=\frac{h(x)}{h_{0}}$ :

$\psi=0, \quad \omega=\frac{\left(1+h^{\prime 2}\right)}{\left(1-h^{\prime 2}\right)} \frac{\partial T^{*}}{\partial S}+\frac{4 h^{\prime}}{\left(1-h^{\prime 2}\right)}\left[\frac{\partial^{2} \psi}{\partial x \partial y}\right]+2 \frac{\partial^{2} \psi}{\partial y^{2}}$

$-\frac{\partial T^{*}}{\partial n}=L T^{*}$

The vorticity at free surface is determined from the equilibrium of tangential stress where $S$ is the tangential direction and $n$ is the normal direction. $L=\alpha h_{0} / k$ is identical to the Biot number where $k$ is the heat conductivity coefficient of liquid and $\alpha$ the heat transfer coefficient at the free surface. The asterisk $(*)$ for the non-dimensional temperature is omitted hereafter.

The free surface shape is determined by the equilibrium of normal stress,

$$
\begin{aligned}
\left(P-P_{\infty}\right)= & \frac{2}{\left(1+h^{\prime 2}\right)}\left[h^{\prime 2} \frac{\partial U}{\partial x}-h^{\prime}\left(\frac{\partial W}{\partial x}+\frac{\partial U}{\partial y}\right)+\frac{\partial W}{\partial y}\right] \\
& -\frac{h^{\prime \prime}}{C a \cdot\left(1+h^{\prime 2}\right)^{3 / 2}}
\end{aligned}
$$

Here $C a=\left|\sigma_{T}\right| \Delta T / \sigma$ is the capillary number. $U$ and $W$ are respectively the non-dimensional velocity components in the directions $x$ and $y$. The free surface at both sidewalls locates at $y=1$ implying the control of free surface at the triple-phase line. Dynamic free-surface deformation during the heating process is neglected.

A hybrid finite element method of fractional steps is adopted in this study to handle the curved free surface [6]. Irregular triangular grids are adopted in this study. The number of cells is $121 \times 21$ in the directions of $x$ and $y$ respectively, and the computation domain is divided in to 4800 triangular elements with 2541 nodes. The liquid layer is initially motionless. The ending condition of calculation is that the evolutions of velocity and temperature at the monitoring points in the liquid layer no longer change with time. The details of the code validation can be found in [7].

\section{Numerical results and discussions}

In the linear stability analysis on infinite liquid layer [1], the marginal stability boundary can be determined through solving an eigenvalue problem involving the parameters of $M a$ and $L$. The critical state is independent of $\mathrm{Pr}$, and a series of convection rolls simultaneously occur and fill up the whole liquid layer. Fig. 1 shows the multi-cellular flow structure evolutions with increasing $\Delta T$ in liquid layers of different volume ratios. Every plot represents the steady state of the convection at a fixed $\Delta T$. $L=0.5$ is adopted at the free surface. Only Marangoni convection is involved in the liquid layer with planar free surface $\left(V / V_{0}=1.0\right)$. With increasing $\Delta T$, the convection rolls initially emerges near the sidewalls. The convection roll number gradually increases until the multi-cellular flow structure fills up the whole liquid layer. This phenomenon is different from the linear stability analysis results. The critical state is defined as the phenomenon that the convection rolls reach the central line of liquid layer which the vertical velocity at the central point $\left(x=9.25, y=0.5 h_{c} / h_{0}\right)$ starts to grow from zero (not shown here), and identically the slope of the effective Marangoni number profile changes sharply (see Fig. 2). At the critical state ( $\Delta T=0.133^{\circ} \mathrm{C}, B=114.02$ ), six pairs of convection rolls fill up the whole liquid layer (see Fig. 1), and the wave number is $\alpha=2.0368$ based on Eq. 3.3 in [2]. Note that the convection rolls develop gradually from sidewalls to the central line of liquid layer. The generation of convection rolls at the central line should overcome the drag force induced by the convection rolls already exist. Therefore the effective critical Marangoni number predicted is larger than $B=98.55$ determined at a state of neutral stability based on [1].

Coupled Marangoni convection and thermocapillary convection occurs in the liquid layers with curved free surface, either concave $\left(V / V_{0}<1.0\right)$ or convex $\left(V / V_{0}>1.0\right)$. As shown in Fig. 1 , even when $\Delta T$ is strictly perpendicular to the liquid layer, thermocapillary 
convection rolls first occurs in the whole liquid layer at any tiny lateral temperature gradient induced by the curved free surface. With increasing $\Delta T$, several pairs of convection rolls arise near the sidewalls for the case of $V / V_{0}=0.9$. On the other hand, the thermocapillary convection rolls persist in the whole liquid layer for the case of $V / V_{0}=1.3$, however, secondary convection rolls emerge in the bulk. At the critical state, the convection rolls start to develop around the central line of liquid layer, and the multi-cellular flow structure occupies the whole liquid layer at the supercritical state finally. Note that the number of convection rolls decreases with increasing volume ratio. Similarly, the critical states can be determined as $\left(\Delta T=0.150^{\circ} \mathrm{C}, \quad B=133.70\right)$ for $V / V_{0}=0.9$ and $\left(\Delta T=0.084{ }^{\circ} \mathrm{C}, B=62.31\right.$ ) for $V / V_{0}=1.3$ respectively through the evolutions of the effective Marangoni number (see Fig. 2).

Fig. 3 shows the marginal instability boundary for the liquid layer of $\operatorname{Pr}=11.6$. The profile consists two branches, one corresponds to $V / V_{0}<1$ and the other corresponds to $V / V_{0}>1$. The critical Marangoni number generally decreases with increasing volume ratio. This tendency may be explained as follows: Since the sidewall height and the aspect ratio of liquid layer are fixed in the present study, smaller volume ratio implies smaller mean depth of liquid layer with more convection rolls in the bulk. Therefore, the generation of convection rolls at the central line of liquid layer with smaller volume ratio has to overcome larger drag force induced by the convection rolls already exist. Correspondingly, the convection rolls in convex liquid layer at the critical state are much less than those in concave free surface (see Fig. 1). On the other hand, with the same configuration, the critical Marangoni number for the concave liquid layers of $\operatorname{Pr}=11.6$ is smaller than that of $\operatorname{Pr}=105.6$ while the critical Marangoni number for the convex liquid layers of $\operatorname{Pr}=11.6$ is generally larger than that of $\operatorname{Pr}=105.6$. This tendency may be explained as follows: The convection roll number decreases with increasing $P r$ in the concave liquid layer, and then the generation of convection rolls at the central line of liquid layer of lower-Pr fluid has to overcome less drag force induced

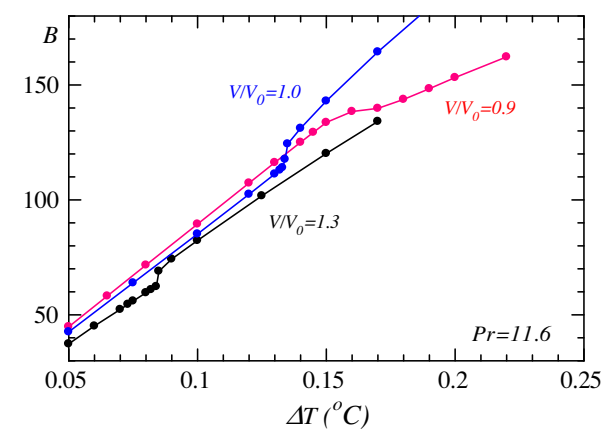

Fig. 2. The effective Marangoni number for the convection in liquid layers $(P r=11.6)$ of $V / V_{0}=0.9,1.0$ and 1.3 as a function of $\Delta T$.

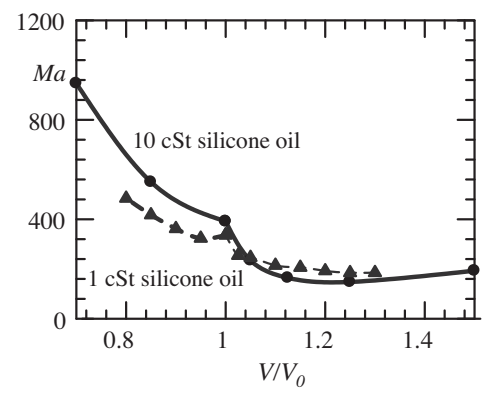

Fig. 3. The marginal stability boundaries for convection in liquid layers ( $P r=11.6$ and $\operatorname{Pr}=105.6$ ) of different volume ratios. by the convection rolls already exist in liquid layer, which corresponds to smaller critical Marangoni number. On the other hand, the number of convection roll number increases with increasing $\mathrm{Pr}$ in the convex liquid layer, and then the generation of convection rolls at the central line of liquid layer of lower-Pr fluid has to overcome larger drag force induced by the convection rolls already exist in liquid layer, which corresponds to larger critical Marangoni number.

An interesting phenomenon is also revealed in liquid layer of $\operatorname{Pr}=11.6$. As shown in Fig. 4, oscillatory multi-cellular coupled convection may occur in the concave liquid layers with small volume ratios, which was not reported in [7]. Particularly, in a narrow range of $0.80 \leqslant V / V_{0} \leqslant 0.85$, the coupled Marangoni convection and thermocapillary convection experiences the regimes of steady multi-cellular convection, oscillatory multi-cellular convection and steady multi-cellular convection in sequence with increasing Marangoni number. The inset of Fig. 4 shows the periodically oscillation of the vertical velocity $W_{c}$ at the central point $(x=9.25$, $y=0.5 h_{c} / h_{0}$ ) of the liquid layer of $V / V_{0}=0.8$. Accordingly, the convection roll number in the liquid layer alternatively changes between 7 pairs and 8 pairs within a period about $1100 \mathrm{~s}$ (not shown here). Fig. 5 shows the propagation of the corresponding temperature disturbance $T$ 'at the free surface. Note that the traveling wave originates from the central point of free surface (the hot spot) and propagates to the sidewalls (the cold spots), which is reverse to the propagation direction of hydrothermal wave which is usually adopted to explain the instability mechanism of the ther-

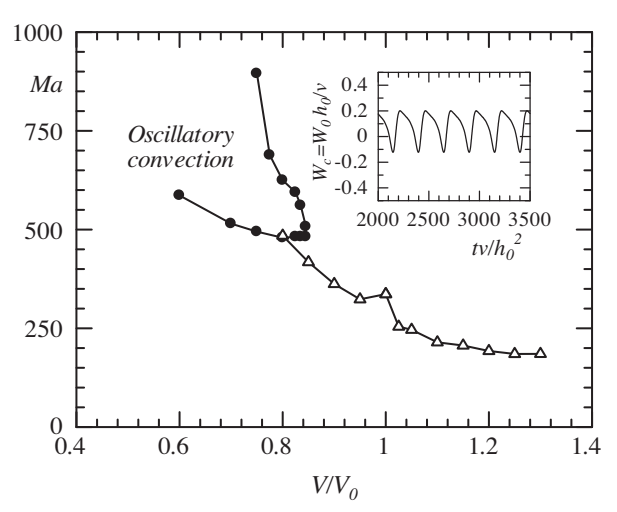

Fig. 4. The marginal stability boundary for oscillatory convection in liquid layers $(\operatorname{Pr}=11.6)$ of different volume ratios. (Inset: the periodically oscillatory vertical velocity $W_{c}$ at the central point $\left(x=9.25, y=0.5 h_{c} / h_{0}\right)$ of the liquid layer of $V /$ $\left.V_{0}=0.8\right)$

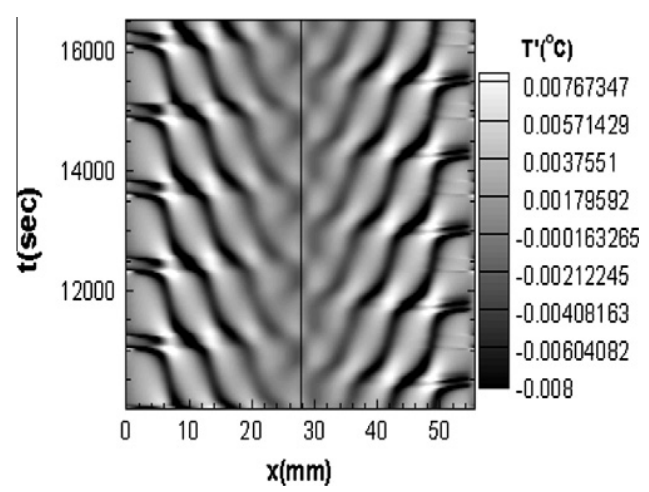

Fig. 5. The propagation of the temperature disturbance $T^{\prime}$ of the oscillatory convection at the free surface of the liquid layer $(P r=11.6)$ of $V / V_{0}=0.8$. 
mocapillary convection in liquid layer and floating half zone of high $\operatorname{Pr}$ fluid.

\section{Conclusions}

Due to the curved free surface, the multi-cellular coupled Marangoni convection and thermocapillary convection is induced by both the vertical and the lateral applied temperature difference in a bottom-heated finite liquid layer $(\mathrm{Pr}=11.6)$ in the microgravity conditions. The critical Marangoni number decreases with increasing volume ratio, and the marginal instability boundary consists two branches related to $V / V_{0}<1$ and $V / V_{0}>1$ respectively. Moreover, oscillatory coupled convection may occur in liquid layers in a narrow range of $0.80 \leqslant V / V_{0} \leqslant 0.85$.

\section{Acknowledgment}

This research is supported by the National Natural Science Foundation of China (Grant Nos. 10872202, 11032011) and the
Knowledge Innovation Project of the Chinese Academy of Sciences (Grant No. KJCX2-YW-L08).

\section{References}

[1] J.R.A. Pearson, On convection cells induced by surface tension, J. Fluid Mech. 4 (1958) 489-500.

[2] S. Rosenblat, G.M. Homsy, S.H. Davis, Nonlinear Marangoni convection in bounded layers. Part 2. Rectangular cylindrical containers, J. Fluid Mech. 120 (1982) 123-138

[3] H.A. Dijkstra, Surface tension driven cellular patterns in three-dimensional boxes-Part II: A bifurcation study, Microgravity Sci. Technol. VIII (2) (1995) 7076.

[4] E.S. Oleg, A.N. Alexander, Thermocapillary flows under an inclined temperature gradient, J. Fluid Mech. 504 (2004) 99-132.

[5] J.M. Zhang, Zh. W Chen, Y.Sh. Li, Y.H. Nie, Three-dimensional double-diffusive Marangoni convection in a cubic cavity with horizontal temperature and concentration gradients, Phys. Rev. E 82 (2010) 066305.

[6] J.H. Wu, A hybrid method of fractional steps with L-stability for numerical modeling of aquatic environments, Adv. Hydrodyn. 1 (3) (1985) 27-36 (in Chinese).

[7] Z.M. Tang, K. Li, W.R. Hu, Influence of free surface curvature of a liquid layer on the critical Marangoni convection, Int. J. Heat Mass Trans. 51 (2008) 5102-5107. 